\title{
REGENERAÇÃO NATURAL EM UM FRAGMENTO DE FLORESTA ESTACIONAL SEMIDECIDUAL EM VIÇOSA, MINAS GERAIS ${ }^{1}$
}

\author{
Luiz Carlos Marangon², João Juares Soares ${ }^{3}$, Ana Lícia Patriota Feliciano² e Carlos Frederico Lins e Silva \\ Brandão $^{4}$
}

\begin{abstract}
RESUMO - O levantamento da regeneração natural das espécies arbóreas em diferentes toposseqüências foi realizado em uma área de aproximadamente 40 ha, localizada no Município de Viçosa, MG, que se encontra nas coordenadas $20^{\circ} 45^{\prime} \mathrm{S}$ e $42^{\circ} 55^{\prime} \mathrm{W}$ e está preservada desde 1965 . A altitude, na área de estudo, apresenta uma cota mínima de $730 \mathrm{~m}$ e uma máxima de $870 \mathrm{~m}$, sendo o solo predominante o Latossolo Vermelho-Amarelo álico, com vegetação de Floresta Estacional Semidecidual. Na amostragem de 1,0 ha foram locadas 40 subparcelas de $25 \mathrm{~m}^{2}$ (5 x 5 m), em 40 parcelas de 10 x 25 m, utilizadas anteriormente em um levantamento fitossociológico das espécies adultas. Essas subparcelas estão distribuídas nas toposseqüências que apresentam áreas diferenciadas e o número de subparcelas foi proporcional à área. Plano (5,07 ha -5 subparcelas), encosta (13,2 ha - 15 subparcelas), ravina (17,28 ha -17 subparcelas) e topo (2,43 ha -3 subparcelas). Os indivíduos amostrados foram incluídos em três classes: a classe 1 contemplou indivíduos com altura $(\mathrm{H}) 1,0 \leq \mathrm{H} \leq 2,0 \mathrm{~m}$; a classe 2 com 2,0 $<\mathrm{H} \leq 3,0 \mathrm{~m}$ e a classe $3 \mathrm{com} \mathrm{H}>3,0 \mathrm{~m}$. O nível de inclusão foi de $\mathrm{CAP}<15,0 \mathrm{~cm}$. Foram amostrados 957 indivíduos, pertencentes a 30 famílias, 72 gêneros e 91 espécies, das quais 31 estavam representadas nas três classes de tamanho. Esse resultado permite dizer que, possivelmente, essas espécies estarão presentes na floresta futura. As espécies com melhor desempenho no que diz respeito à regeneração natural total por classe de altura foram: Psychotria sessilis, Siparuna arianeae, Anadenathera macrocarpa, Nectandra saligna, Piptadenia gonoacantha, Nectandra rigida, Bauhinia forficata, Psycotria carthagenensis, Miconia pusilliflora e Mollinedia floribunda. As espécies Psycotria sessilis e Siparuna arianeae, com 254 e 76 indivíduos, respectivamente, chamam a atenção por sua presença agressiva no processo de regeneração, por serem típicas do sub-bosque. Pela análise de correspondência realizada nas toposseqüências, foi visto que a ravina e o plano tiveram maior similaridade florística do que as demais, possivelmente pela semelhança, no que diz respeito ao ambiente.
\end{abstract}

Palavras-chave: Toposseqüências, análise de correspondência e florística.

\section{NATURAL REGENERATION IN A FRAGMENT OF A SEASONAL SEMIDECIDUOUS FOREST IN VIÇOSA - MINAS GERAIS}

\begin{abstract}
Natural regeneration of tree species at different toposequences was assessed in an area of 40 ha in Viçosa-MG. ( $20^{\circ} 45^{\prime} \mathrm{S}$ and $42^{\circ} 55^{\prime} \mathrm{W}$ ), preserved since 1965, with minimum altitude of $730 \mathrm{~m}$ and maximum of $870 \mathrm{~m}$. The soil was classified as Red-yellow alico Latisol and the vegetation was characterized as semideciduous forest.The 1.0 samplings consisted of 40 sub-units (samples with $5 \times 5 \mathrm{~m}$ ). These sub-units were inserted in the middle samples $(10 \times 25 \mathrm{~m})$.previously evaluated the phyto-sociology of the tree community distributed in the toposequence's different: plan (5.07 ha - 5 sub-units), lean (13.2 ha - 15 sub-units), ravine (17.28 ha-17 sub-units) and top (2.43 ha-3 sub-units). Was developed with heights divided in three categories ( 1 -individuals with minimum height $1 \leq 2 \mathrm{~m} ; 2$-individuals with minimum heights $2 \leq 3 \mathrm{~m}$; 3 -individuals
\end{abstract}

\footnotetext{
${ }^{1}$ Recebido em 20.12.2006 e aceito para publicação em 18.12.2007.

${ }^{2}$ Professor Departamento de Ciência Florestal - UFRPE. Recife-PE. E-mail : <marangon@dcfl.ufrpe.br>e <licia@dcfl.ufrpe.br>.

${ }^{3}$ Professor Programa de Pós-Graduação em Ecologia e Recursos Naturais - UFSCAR. E-mail : <juares@power.ufscar.br>.

${ }^{4}$ Programa de Pós-Graduação em Ciências Florestais da UFRPE. E-mail : <cflsbrandao@hotmail.com>.
} 
with heigts $>3 \mathrm{~m}$ ). The inclusion level was of CAP $<15,0 \mathrm{~cm} .957$ individuals were observed, belonging to 30 families, 72 genery and 91 species. The regeneration presented 31 species in the three size classes. This result allows saying that possibly, these species, will be present in the future forest. The species with better acting in the total natural regeneration for height class were: Psychotria sessilis, Siparuna arianeae, Anadenathera macrocarpa, Nectandra saligna, Piptadenia gonoacantha, Nectandra rigida, Bauhinia forficata, Psycotria carthagenensis, Miconia pusilliflora and Mollinedia floribunda. These species had presented efficient in what concerns the succession process in the area. Psycotria sessilis and Siparuna arianeae, with 254 and 76 individuals respectively, get the attention for their aggressive presence in the regeneration process, for they be typical of the sub-forest. For the analysis of Correspondence carried through in the toposequence, it was since the ravine and plan had had greater floristic similarity of what excessively.

Keywords: Toposequence, correspondence analysis and Floristics.

\section{INTRODUÇÃO}

Por ser um ecossistema complexo, a floresta tropical sempre é um desafio para a ciência florestal. O conhecimento desse recurso é uma necessidade, visto que, a cada momento, intervenções sucessivas acontecem, sem a mínima preocupação com a conservação desse recurso natural.

De modo geral, os remanescentes desse bioma encontram-se em estádio de sucessão natural secundária, fragmentados, alterados e empobrecidos em sua composição florística original. Ainda que sofrendo intensa perturbação, esses povoamentos florestais nativos são um valioso recurso natural renovável, passível de utilização pelas gerações presentes e futuras. Entretanto, esse recurso aumentará se for permitida a regeneração do estoque e decrescerá se esta não for permitida. Portanto, os conhecimentos da regeneração natural e dos processos de dinâmica de sucessão, crescimento e produção são fundamentais para a utilização desses recursos naturais (SOUZA et al., 2002).

A regeneração natural decorre da interação de processos naturais de restabelecimento do ecossistema florestal, é, portanto, parte do ciclo de crescimento da floresta e refere-se às fases iniciais de seu estabelecimento e desenvolvimento. O estudo da regeneração natural permite a realização de previsões sobre o comportamento e desenvolvimento futuro da floresta, pois fornece a relação e a quantidade de espécies que constitui o seu estoque, bem como suas dimensões e distribuição na área (GAMA et al., 2002).

Esse tipo de processo é parte do complexo biológico ativo das florestas tropicais que formam, desenvolvem e mantém as fitofisionomias. Jardim et al. (1993), mencionam que as principais mudanças estruturais e florísticas numa capoeira ocorrem durante seus primeiros dez anos, ao fim dos quais a fisionomia da vegetação é semelhante à da floresta madura. Para Negrelle (1995), o dossel atual de uma floresta é composto por árvores que provavelmente iniciaram seu ciclo vital muitos anos atrás e que comportam abaixo de si os sobreviventes de sucessivos períodos de regeneração, a partir de sementes ou de outros mecanismos.

As florestas estacionais semidecíduas do Estado de Minas Gerais hoje são matas secundárias oriundas de regeneração natural. Fica claro, nesse caso, que a compreensão da regeneração e da dinâmica das espécies arbóreas desse ecossistema é de fundamental importância para o desenvolvimento de técnicas de manejo adequadas para a manutenção da diversidade florística dessas áreas.

De maneira geral, a floresta tropical apresenta composição florística muito diferente entre a fração adulta ou dossel e a fração do sub-bosque (UHL e MURPHY, 1981; JARDIM e HOSOKAWA, 1986), devido a dois fatores: o componente herbáceo-arbustivo, que caracteristicamente não atinge grandes tamanhos, no máximo alcançando o estrato inferior e o grande número de espécies heliófilas, com pouca ou nenhuma regeneração, mas que são bem representadas na massa do dossel (JARDIM et al., 1993).

Fica evidente que estudos de regeneração natural devem ser implementados nas diversas fitofisionomias da floresta tropical brasileira, em função da necessidade de informações básicas desses ecossistemas. Sendo assim, esse estudo tem, como objetivo, a verificação da composição florística que se encontra regenerando 
na Mata da Pedreira, no sentido de melhor conhecer o comportamento dessas espécies que formam essa comunidade.

\section{MATERIAL E MÉTODOS}

A Mata da Pedreira (Figura 1), se localiza no município de Viçosa, a $6 \mathrm{~km}$ da cidade, nas coordenadas $20^{\circ} 45^{\prime}$ ' S e $42^{\circ} 55^{\prime}$ ' W, no norte da Zona da Mata Mineira (MARISCAL FLORES, 1993). A altitude varia de 600 a $800 \mathrm{~m}$, podendo ser encontradas altitudes superiores a 800 m nos topos dos morros da região (MEIRA-NETO, 1997). A área estudada apresenta, em termos de altitude, uma cota mínima de 730 m e uma cota máxima de 870 m. Segundo a classificação de Köpen, o clima é do tipo Cwb, mesotérmico, com verões chuvosos, invernos frios e secos. A precipitação média, nos últimos 30 anos, foi de aproximadamente $1221 \mathrm{~mm}$ (MARANGON, 2003). O solo predominante é Latossolo Vermelho-Amarelo álico. A vegetação da região é Floresta Estacional Semidecidual e o fragmento estudado é uma floresta secundária preservada desde 1965.

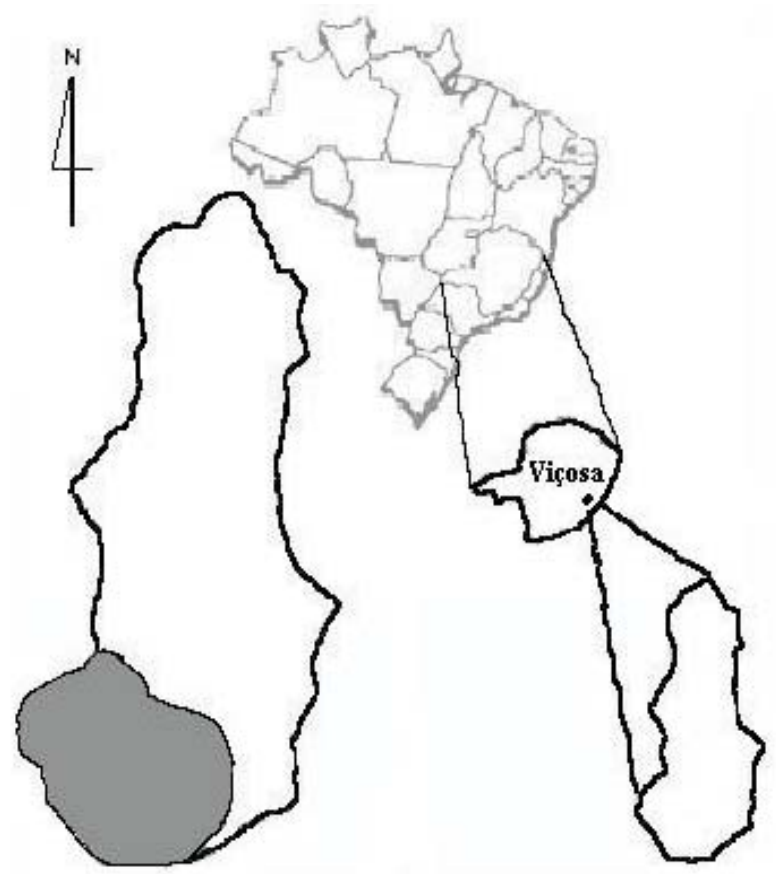

Figura 1 - Localização da Mata da Pedreira, situada na Estação Experimental Mata do Paraíso do Departamento de Engenharia Florestal da UFV, Viçosa, MG.

Figure 1 - Mata da Pedreira location at Mata do Paraiso Experimental Station of the Department of Forestry of the UFV, Viçosa, MG.
Estimou-se a regeneração natural das espécies com base na metodologia empregada por Finol (1971) e modificada por Volpato (1994). Nas 40 parcelas de 10 x 25 m utilizadas na abordagem de indivíduos adultos, para o estudo da estrutura fitossociológica, locaramse 40 sub-parcelas de $25 \mathrm{~m}^{2}$ ( $5 \mathrm{~m}$ x $5 \mathrm{~m}$ ). O número de parcelas por toposseqüência, foram definidas proporcionalmente a área de cada uma delas, ficando assim implantadas: Plano (5,07 ha -5 sub-parcelas), encosta (13,2 ha - 15 sub-parcelas), ravina $(17,28$ ha - 17 sub-parcelas) e topo (2,43 ha -3 sub-parcelas). Os indivíduos foram etiquetados e com a ajuda de uma trena de bolso de 5m, mediu-se a altura. Quando necessário estimava-se o restante com a tesoura de alta poda com módulos de 2,0 metros.

Os indivíduos foram agrupados nas seguintes classes de altura: classe 1 contemplou indivíduos com altura (H) 1,0 $\leq \mathrm{H} \leq 2,0 \mathrm{~m}$; a classe $2 \operatorname{com} 2,0<\mathrm{H} \leq$ $3,0 \mathrm{~m}$ e a classe $3 \mathrm{com} \mathrm{H}>3,0 \mathrm{~m}$ e CAP menor que $15,0 \mathrm{~cm}$. Optou-se pela altura mínima de $1,0 \mathrm{~m}$ para análise da regeneração de espécies arbóreas, visto que, nessa altura, as espécies apresentam uma melhor definição da sua caracterização morfológica, permitindo identificação mais confiável. Em alturas inferiores a essa estabelecida, é difícil definir grupos, como, por exemplo, lianas de árvores, muitas menos as espécies que apresentam variações morfológicas ao passarem de plântulas para muda. Para cada espécie estimaramse os parâmetros: freqüência e densidade absoluta e relativa em cada classe de altura pré-estabelecida. Com base nesses cálculos, obteve-se a estimativa da regeneração natural por classe de altura.

Calculado o índice de regeneração por classe de altura para cada espécie, o passo seguinte foi o cálculo da estimativa da regeneração total dentro das classes por espécie, utilizando-se da soma dos índices de regeneração natural por classe de altura.

Para serem feitas comparações florísticas entre as toposseqüências analisadas, foi obtida uma matriz de presença e ausência das espécies em acordo com sua toposseqüiência, e para a ordenação das mesmas foi usado o método de média recíproca (RA) ou análise de correspondência (CA), utilizando o programa Pc-Ord for Windows versão 4.14 (MCCUNE e MEFFORD, 1999).

\section{RESULTADOS E DISCUSSÃO}

Nas quarenta sub-parcelas amostradas encontraramse 957 indivíduos, pertencentes a 30 famílias, 72 gêneros

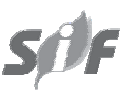

R. Árvore, Viçosa-MG, v.32, n.1, p.183-191, 2008 
e 91 espécies no levantamento da regeneração na Mata da Pedreira. As estimativas da regeneração natural por classe de altura encontram-se na Tabela 1. As dez espécies de maiores valores de regeneração natural total (RNT) foram as seguintes: Psychotria sessilis, Siparuna arianae, Anadenathera macrocarpa, Nectandra saligna, Piptadenia gonoacatha, Nectandra rigida, Bauhinia forficata, Psycotria carthagenensis, Miconia pusiliflora e Mollinedia floribunda.

Em termos de soma total da regeneração por classe de altura (RNC) as porcentagens estão assim distribuídas por classe: espécies que ocorrem na classe 3, contribuíram com 23,01\% , espécies que ocorrem na classe 2 , contribuíram com $22,68 \%$ e espécies que ocorrem na classe 1, contribuíram com 54,31\% (Tabela 1).

Das 91 espécies amostradas, 32 ocorrem nas três classes de regeneração por classe de altura ( $\mathrm{RNC}_{1}$,
$\mathrm{RNC}_{2}, \mathrm{RNC}_{3}$ ), 22, em duas classes ( $\mathrm{RNC}_{1}$ e $\mathrm{RNC}_{2}$ ou $\mathrm{RNC}_{1}$ e $\mathrm{RNC}_{3}$ ou $\mathrm{RNC}_{2}$ e $\mathrm{RNC}_{3}$ ) e 37 em uma classe ( $\mathrm{RNC}_{1}$ ou $\mathrm{RNC}_{2}$ ou RNC $\mathrm{RN}_{3}$. Segundo Volpato (1994), a presença de uma espécie na classe de menor tamanho, com elevada densidade, apresenta índice de regeneração elevado. Os resultados, no entanto, devem ser analisados com cautela, pois a mesma pode desaparecer ainda nessa fase inicial do desenvolvimento.

Espécies como Guapira opposita, Xylopia sericea, Platypodium elegans, Trichilia hirta e Pseudobombax grandiflorum ocorreram somente na classe de menor tamanho, com densidade proporcional alta, e confirmam a expectativa de estarem presentes na floresta futura, pois as mesmas estão presentes na amostragem das adultas. A espécie Pseudobombax grandiflorum aparece entre as dez espécies de maior valor de VI (MARANGON, 1999).

Tabela 1 - Espécies amostradas nas subparcelas de regeneração na Mata da Pedreira, por classe de altura (RNC) e estimativa da Regeneração Natural Total (RNT), onde RNC1 = Regeneração Natural na Classe 1; RNC2 = Regeneração Natural na Classe 2; e RNC3 = Regeneração Natural na Classe 3

Table 1 - Species obtained in the regeneration split-plots in Mata da Pedreira by height class (RNC) and estimate of the Total Natural Regeneration $(T N R)$, where RNC1 = Natural Regeneration in Class 1; RNC2 = Natural Regeneration in Class 2; RNC3 = Natural Regeneration in Class 3

\begin{tabular}{|c|c|c|c|c|}
\hline Espécies & RNC1(\%) & RNC2(\%) & RNC3(\%) & $\mathrm{RNT}(\%)$ \\
\hline Psychotria sessilis (Vell.) Müll. Arg. & 11,05 & 4,47 & 3,8 & 19,32 \\
\hline Siparuna arianeae V. Pereira & 3,6 & 1,62 & 2,23 & 7,45 \\
\hline Anadenanthera macrocarpa (Benth.) Brenan & 2,95 & 1,76 & 1,05 & 5,76 \\
\hline Nectandra saligna Nees & 3,03 & 1,67 & 0,8 & 5,5 \\
\hline Piptadenia gonoacantha (Mart.) J.F. Macbr. & 3,05 & 1,21 & 0,4 & 4,66 \\
\hline Nectandra rigida (Kunth) Nees & 2,83 & 0,81 & 1,02 & 4,66 \\
\hline Bauhinia forficata Link & 1,1 & 0,96 & 1,26 & 3,32 \\
\hline Psychotria carthagenensis Jacq. & 1,78 & 1,01 & 0,15 & 2,94 \\
\hline Miconia pusilliflora Beurl. & 1,16 & 0,8 & 0,75 & 2,71 \\
\hline Mollinedia floribunda Tul. & 1,38 & 0,81 & 0,15 & 2,34 \\
\hline Myrcia rostrata DC. & 1,31 & 0,35 & 0,3 & 1,96 \\
\hline Inga cylindrica (Vell.) Mart. & 1,28 & 0,15 & 0,45 & 1,88 \\
\hline Endlicheria paniculata (Spreng.) J.F. Macbr. & 0,9 & 0,3 & 0,45 & 1,65 \\
\hline Machaerium triste Vogel & 1,1 & 0,3 & 0,15 & 1,55 \\
\hline Brosimum guianense (Aubl.) Huber & 0,82 & 0,15 & 0,5 & 1,47 \\
\hline Trichilia pallida Sw. & 0,55 & 0,4 & 0,31 & 1,26 \\
\hline Luehea speciosa Willd. & 0,7 & 0,2 & 0,3 & 1,2 \\
\hline Casearia gossypiosperma Briq. & 0,15 & 0,3 & 0,6 & 1,05 \\
\hline Nectandra reticulata (Ruiz \& Pav.) Mez & 0,71 & 0 & 0,3 & 1,01 \\
\hline Apuleia leiocarpa (Vogel) J.F. Macbr. & 0,35 & 0,15 & 0,5 & 1 \\
\hline Anadenanthera colubrina (Vell.) Brenan & 0,55 & 0,3 & 0,15 & 1 \\
\hline Sorocea guilleminiana Gaudich. & 0,55 & 0,45 & 0 & 1 \\
\hline Prunus sellowii Koehne & 0,8 & 0,15 & 0 & 0,95 \\
\hline Dalbergia nigra (Vell.) Allemao ex Benth. & 0,15 & 0 & 0,78 & 0,93 \\
\hline
\end{tabular}

R. Árvore, Viçosa-MG, v.32, n.1, p.183-191, 2008 
Tabela 1 - Cont.

Table 1 - Cont.

\begin{tabular}{|c|c|c|c|c|}
\hline Espécies & RNC1(\%) & RNC2(\%) & RNC3(\%) & $\mathrm{RNT}(\%)$ \\
\hline Machaerium nyctitans (Vell.) Benth. & 0,43 & 0 & 0,38 & 0,81 \\
\hline Randia armata (Sw.) DC. & 0,57 & 0,24 & 0 & 0,81 \\
\hline Matayba elaeagnoides Radlk. & 0,3 & 0 & 0,46 & 0,76 \\
\hline Sparattosperma leucanthum (Vell.) K. Schum. & 0,38 & 0,19 & 0,19 & 0,76 \\
\hline Cupania vernalis Cambess. & 0,38 & 0,19 & 0,19 & 0,76 \\
\hline Casearia arborea (Rich.) Urb. & 0,3 & 0,15 & 0,3 & 0,75 \\
\hline Coutarea speciosa Aubl. & 0,15 & 0,45 & 0,15 & 0,75 \\
\hline Vernonia diffusa Less. & 0,19 & 0,19 & 0,37 & 0,75 \\
\hline Lonchocarpus muehlbergianus fo. angustifoliolata Hassl. & 0,19 & 0,19 & 0,37 & 0,75 \\
\hline Dictyoloma vandellianum A.H.L. Juss. & 0,19 & 0,19 & 0,37 & 0,75 \\
\hline Ocotea martiana (Meisn.) Mez & 0,27 & 0,15 & 0,3 & 0,72 \\
\hline Casearia sylvestris Sw. & 0,3 & 0,2 & 0,15 & 0,65 \\
\hline Ixora bahiensis Benth. & 0,35 & 0,15 & 0,15 & 0,65 \\
\hline Rheedia spruceana Engl. & 0,15 & 0,3 & 0,15 & 0,6 \\
\hline Lacistema pubescens Mart. & 0,45 & 0 & 0,15 & 0,6 \\
\hline Tapirira guianensis Aubl. & 0,45 & 0 & 0,15 & 0,6 \\
\hline Astronium fraxinifolium Schott ex Spreng. & 0,4 & 0 & 0,15 & 0,55 \\
\hline Alchornea glandulosa Poepp. & 0,2 & 0 & 0,3 & 0,5 \\
\hline Jacaranda macrantha Cham. & 0,15 & 0,15 & 0,15 & 0,45 \\
\hline Rollinia sylvatica (A. St.-Hil.) Martius & 0,15 & 0,3 & 0 & 0,45 \\
\hline Myrcia rufula Miq. & 0,3 & 0,15 & 0 & 0,45 \\
\hline Cabralea canjerana (Vell.) Mart. & 0,3 & 0 & 0,15 & 0,45 \\
\hline Erythroxylum pelleterianum A. St.-Hil. & 0 & 0,15 & 0,3 & 0,45 \\
\hline Annona cacans Warm. & 0,3 & 0,15 & 0 & 0,45 \\
\hline Croton piptocalyx Müll. Arg. & 0 & 0 & 0,45 & 0,45 \\
\hline Guapira opposita (Vell.) Reitz & 0,4 & 0 & 0 & 0,4 \\
\hline Syagrus romanzoffiana (Cham.) Glassman & 0,35 & 0 & 0 & 0,35 \\
\hline Seguieria langsdorffii Moq. & 0,15 & 0,2 & 0 & 0,35 \\
\hline Virola oleifera (Schott) A.C. Sm. & 0,15 & 0,15 & 0 & 0,3 \\
\hline Miconia cinnamomifolia (DC.) Naudin & 0,15 & 0 & 0,15 & 0,3 \\
\hline Zanthoxylum rhoifolium Lam. & 0,15 & 0 & 0,15 & 0,3 \\
\hline Cybistax antisyphilitica (Mart.) Mart. & 0,15 & 0,15 & 0 & 0,3 \\
\hline Cestrum sp. & 0,15 & 0,15 & 0 & 0,3 \\
\hline Xylopia sericea A. St.-Hil. & 0,3 & 0 & 0 & 0,3 \\
\hline Platypodium elegans Vogel & 0,3 & 0 & 0 & 0,3 \\
\hline Trichilia hirta L. & 0,3 & 0 & 0 & 0,3 \\
\hline Albizia polycephala (Benth.) Killip ex Record & 0,3 & 0 & 0 & 0,3 \\
\hline Pseudobombax grandiflorum (Cav.) A. Robyns & 0,3 & 0 & 0 & 0,3 \\
\hline Myrciaria sp. & 0,3 & 0 & 0 & 0,3 \\
\hline Piptadenia adiantoides (Spreng.) J.F. Macbr. & 0 & 0 & 0,3 & 0,3 \\
\hline Machaerium stipitatum (DC.) Vogel & 0,2 & 0 & 0 & 0,2 \\
\hline Allophylus edulis (A. St.-Hil., Cambess. \& A. Juss.) Radlk. & 0 & 0 & 0,15 & 0,15 \\
\hline Xylosma prockia (Turcz.) Turcz. & 0,15 & 0 & 0 & 0,15 \\
\hline Guarea pendula Ramalho Pinheiro \& Pennington & 0,15 & 0 & 0 & 0,15 \\
\hline Sapium glandulatum (Vell.) Pax & 0 & 0 & 0,15 & 0,15 \\
\hline Ocotea laxa (Nees) Mez & 0,15 & 0 & 0 & 0,15 \\
\hline Ocotea corymbosa (Meisn.) Mez & 0 & 0,15 & 0 & 0,15 \\
\hline Matayba leucodictya Radlk. & 0,15 & 0 & 0 & 0,15 \\
\hline Campomanesia xanthocarpa (Mart.) O. Berg & 0 & 0,15 & 0 & 0,15 \\
\hline Ixora warmingii Müll. Arg. & 0 & 0 & 0,15 & 0,15 \\
\hline Copaifera langsdorffii Desf. & 0 & 0 & 0,15 & 0,15 \\
\hline
\end{tabular}

R. Árvore, Viçosa-MG, v.32, n.1, p.183-191, 2008 
Tabela 1 - Cont.

Table 1 - Cont.

\begin{tabular}{lcccc}
\hline Espécies & RNC1(\%) & RNC2(\%) & RNC3(\%) & RNT(\%) \\
\hline Vismia guianensis (Aubl.) Choisy & 0 & 0 & 0,15 & 0,15 \\
Alibertia concolor (Cham.) K. Schum. & 0 & 0 & 0,15 & 0,15 \\
Solanum leucodendron Sendtn. & 0 & 0 & 0,15 & 0,15 \\
Marlierea sp. & 0,15 & 0 & 0 & 0,15 \\
Guatteria villosissima St.Hilaire & 0,15 & 0 & 0 & 0,15 \\
Himatanthus phagedaenicus (Mart.) Woodson & 0,15 & 0 & 0 & 0,15 \\
Amaioua guianensis Aubl. & 0,15 & 0 & 0 & 0,15 \\
Lamanonia tomentosa (Cambess.) Kuntze & 0,15 & 0 & 0 & 0,15 \\
Guarea macrophylla Vahl & 0,15 & 0 & 0 & 0,15 \\
Eugenia dodonnaefolia var. grandifolia O. Berg & 0 & 0,15 & 0 & 0,15 \\
Bauhinia sp. & 0,15 & 0 & 0 & 0,15 \\
Carpotroche brasiliensis (Raddi) Endl. & 0,15 & 0 & 0 & 0,15 \\
Swartzia myrtifolia Sm. & 0,15 & 0 & 0 & 0,15 \\
Cordia sericicalyx A. DC. & 0,15 & 0 & 0 & 0,15 \\
Aegiphila sellowiana Cham. & 0 & 0 & 0,15 & 0,15 \\
Xylopia brasiliensis Spreng. & 0,15 & 0 & 0 & 0,15 \\
\hline Total & 54,31 & 22,68 & 23,01 & 100 \\
\hline
\end{tabular}

Os valores obtidos pelo índice de regeneração natural total (RNT) no povoamento estudado variaram de 19,32\% a $0,15 \%$. Para a mesma tipologia florestal do presente estudo, ou seja, Floresta Estacional Semidecidual, Volpato (1994) obteve índices de regeneração natural total (RNT), com valores entre $25,79 \%$ e $0,40 \%$. Citadini-Zanette (1995), para um remanescente de Mata Atlântica em Orleans, SC, obteve valores de índice de regeneração total (RNT) numa amplitude de 8,15\% a 0,04\% e Negrelle (1995), também em Mata Atlântica, no nordeste catarinense, obteve valores para o índice de regeneração total (RNT) de $18,40 \%$ a $0,09 \%$.

Ao se analisar a composição da vegetação arbórea da área de estudo verifica-se que as espécies com grande número de indivíduos nas classes menores de diâmetro tendem a estar presentes na floresta futura daquele local. Essa afirmativa é constatada quando se observam na regeneração os resultados e se verifica a presença dessas espécies em destaque no povoamento. Fica evidente, no entanto, que a Mata da Pedreira está desenvolvendo seu processo sucessional de forma eficiente e garantindo a fitofisionomia da região, ou seja, a Floresta Estacional Semidecidual. Estas afirmativas estão de acordo com Citadini-Zanette (1995), quando a autora afirma que as espécies que ocorrem em todas as classes de altura (RNC) teoricamente possuiriam maior potencial para participar da composição futura da floresta, ou seja, são as que melhor conseguem estabelecer-se na floresta futura.

R. Árvore, Viçosa-MG, v.32, n.1, p.183-191, 2008
A Figura 2 demonstra o desempenho das espécies em regeneração na área estudada, em termos de número de indivíduos por espécie. Foram consideradas aquelas que apresentavam um número mínimo de 15 ou mais indivíduos.

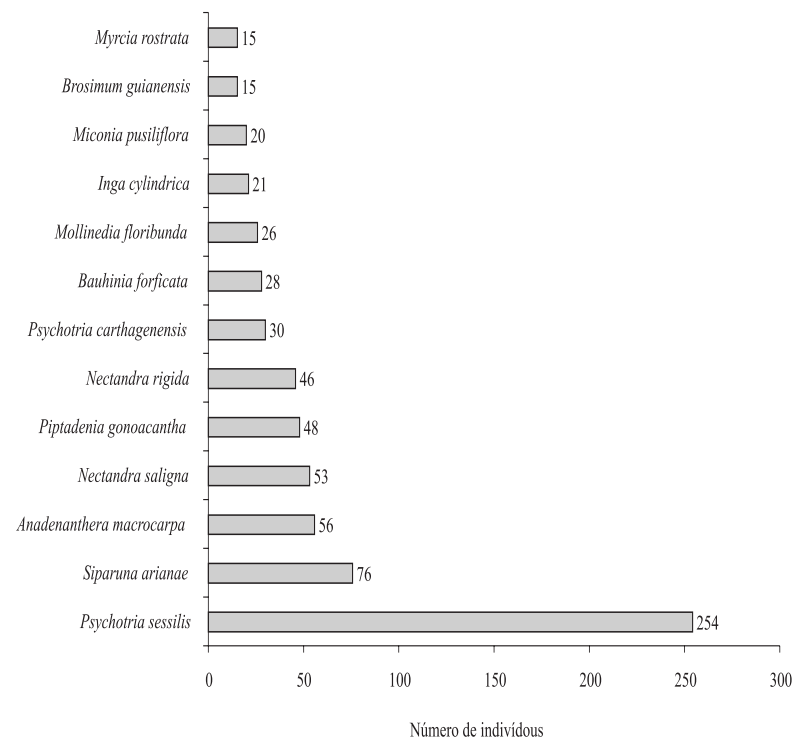

Figura 2-Espécies, em regeneração, com número de indivíduos igual ou superior a 15 na Mata da Pedreira em Viçosa, MG

Figure 2 - Species, under regeneration, number of individuals equal or superior to 15 in Mata da Pedreira in Viçosa, $M G$. 
Em termos de abundância, o destaque fica para Psycotria sessilis, com maior número de indivíduos. É uma espécie secundária inicial, típica de sub-bosque que normalmente atinge 7,0 a 8,0 m de altura e diâmetro pouco desenvolvido, em torno de no máximo $25,0 \mathrm{~cm}$ (MARANGON, 1999). Essa espécie é seguida por Siparuna arianae, que é também uma espécie secundária inicial cujas dimensões estão próximas de Psychotria sessilis, são sempre agressivas em termos de ocupação de espaço, principalmente, em dossel aberto onde as plantas apresentam dimensões menores e um número grande de indivíduos. Em dossel fechado, onde o número de indivíduos é menor, o porte é maior, Anadenanthera macrocarpa, Nectandra saligna e Piptadenia gonoacantha apresentaram comportamento diferente das duas anteriores. São também agressivas, principalmente a primeira e a terceira o mesmo não acontecendo com a segunda, porém apresenta uma regeneração eficiente. Anadenanthera macrocarpa e Piptadenia gonoachanta ocupam espaço rapidamente em início de sucessão e permanecem por um bom tempo nos estratos superiores, onde apresentam indivíduos com dimensões de 10,0 a 20,0 m de altura e diâmetro que pode chegar a 80,0 cm (MARANGON, 1999).

As dez espécies com maiores índices de regeneração total (RNT) contribuem com 58,66\% dos valores desse índice e estão, assim distribuídas: Psychotria sessilis (19,32\%), Siparuna arianae (7,45\%), Anadenanthera macrocarpa (5,76\%), Nectandra saligna (5,50\%), Piptadenia gonoacantha (4,66\%), Nectandra rigida (4,66\%), Bauhinia forficata (3,32\%), Psycotria carthagenensis (2,94\%), Miconia pusiliflora (2,71\%) e Mollinedia floribunda (2,34\%) (Figura 3). Essas espécies possuem uma boa capacidade de regeneração pelo desempenho mostrado. Resultado semelhante foi encontrado por Volpato (1994), em Viçosa, Minas Gerais, apesar de utilizar metodologia diferente em relação ao presente trabalho.

É visto que determinados grupos de espécies mostraram-se indiferentes ao ocorrer nesse ou naquele ambiente. Outras preferem somente ravina ou somente encosta ou somente plano ou então topo. Algumas espécies preferem a combinação de toposseqüências, podendo ocorrer em duas e até em todas. Pela relação espacial da presença ou ausência dessas espécies nas diferentes toposseqüências analisadas através da Análise de Correspondência (CA), demonstrou autovalores bem mais elevados no primeiro eixo de ordenação $(0,448)$ que no segundo (0,374) (Figura 4). De acordo com Felfilli (1998), autovalor é um coeficiente de variação do desvio padrão individual dos perfis de abundância das espécies. Autovalores que sejam maiores que 0,3 , podem ser consideradas divisões mais fortes e significativas. A partir desses dados, verifica-se que há formação de apenas um grupo formado pelas toposseqüências ravina e plano, que contem espécies com maior correlação entre si como Albizia polycephala, Guapira opposita, Jacaranda macrantha, Mollinedia floribunda e Seguieria langsdorffii, onde foram responsáveis pelo seu agrupamento. As demais espécies tiveram maior correlação com as outras toposseqüências.

Da amostragem realizada, foi visto que seis espécies estiveram presentes em todas as toposseqüências, são elas: Anadenanthera macrocarpa, Myrcia rostrata, Nectandra rígida, Ocotea martiana, Piptadenia gonoacantha e Siparuna guianensis.

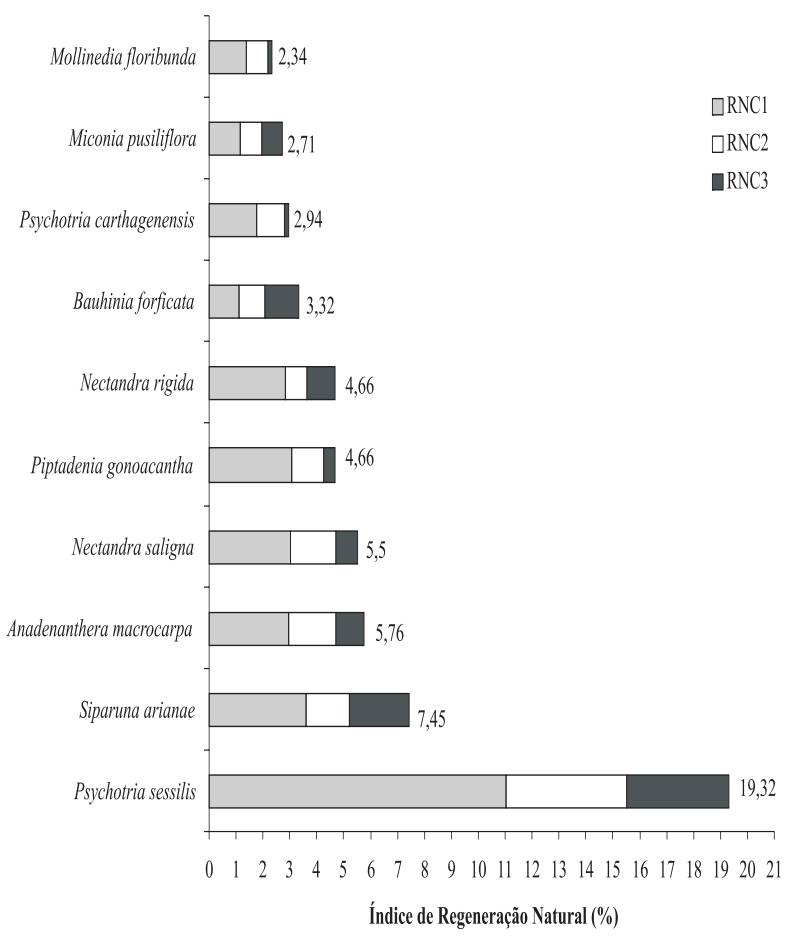

Figura 3 - As 10 espécies com maior índice de regeneração natural total (RNT) e o índice de regeneração natural nas classes de altura $\left(\mathrm{RNC}_{1}, \mathrm{RNC}_{2} \mathrm{e} \mathrm{RNC}_{3}\right)$ amostradas na Mata da Pedreira, em Viçosa, MG.

Figure 3 - The ten species with the highest index of total natural regeneration (TNR) and index of natural regeneration in the height classes (TNR1, TNR2 and TNR3) sampled in Mata Pedreira, Viçosa, MG.

R. Árvore, Viçosa-MG, v.32, n.1, p.183-191, 2008 


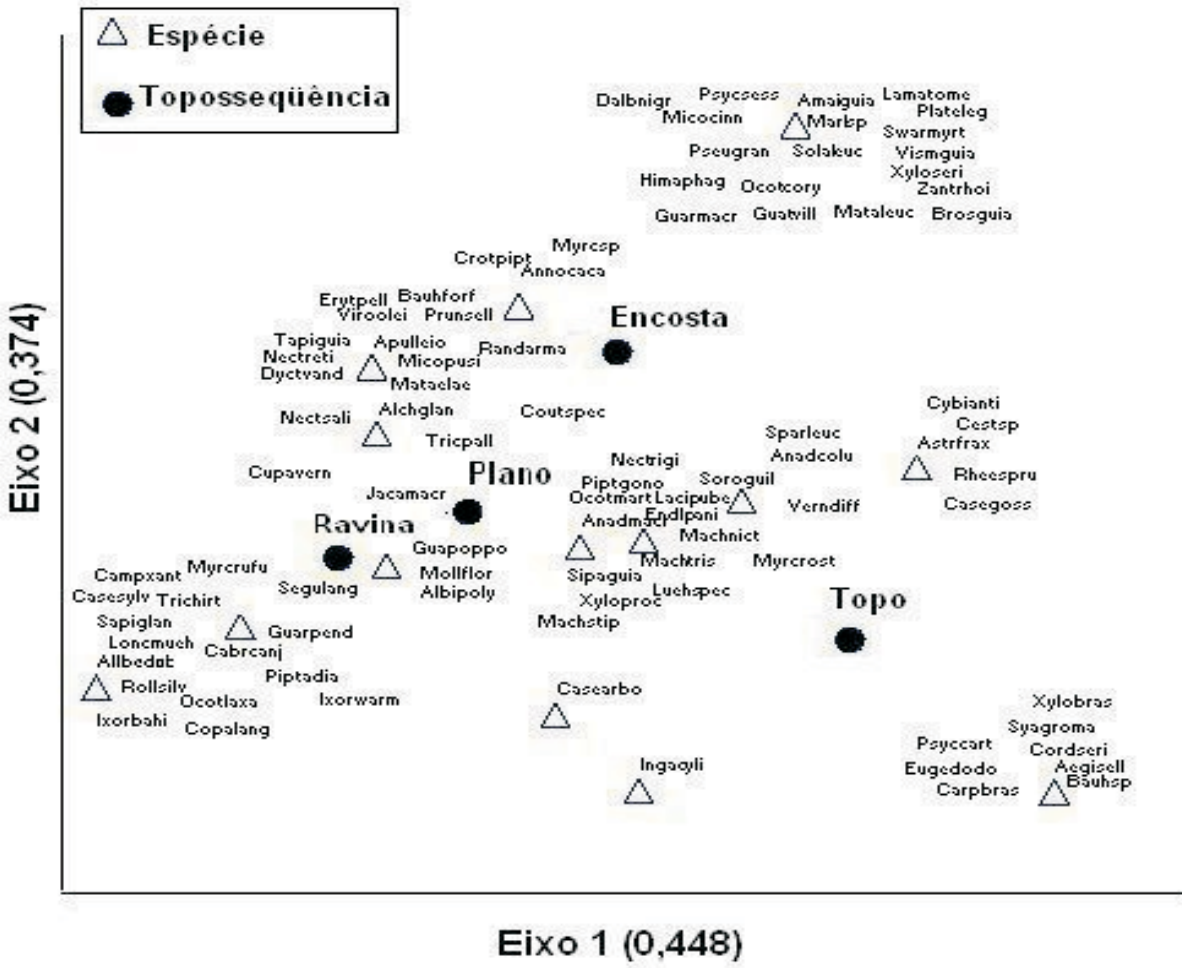

Figura 4 - Ordenação das toposseqüências, através da análise de correspondência (CA), pela presença e ausência das espécies arbóreas encontradas na regeneração na Mata da Pedreira em Viçosa, MG.

Figure 4 - Toposequence ordinance by correspondence analyses (CA), based on the presence and absence of the tree species found in the regeneration of Mata da Pedreira, Viçosa, MG.

A regeneração da Mata da Pedreira apresenta em sua dinâmica, tendências bem claras de que a floresta futura pode apresentar preferências para ocorrer em toposseqüências distintas. As noventa e uma espécies presentes na amostragem da regeneração indicam que isso pode acontecer. Porém, esse comportamento deve ser analisado com cautela em função da amostragem de cada toposseqüência realizada no presente estudo. Trabalhos futuros poderão confirmar ou não essa tendência, visto que são hipóteses e merecem ser certificadas, principalmente, quando se pretende conhecer a dinâmica de sucessão da Floresta Estacional Semidecidual da Zona da Mata de Minas Gerais.

\section{CONCLUSÃO}

Pela análise da composição da vegetação arbórea da área de estudo, é visto que as espécies com grande número de indivíduos nas classes menores de diâmetro tendem a estar presente na floresta futura daquele local. Fica evidente, no entanto, que a Mata da Pedreira está desenvolvendo seu processo sucessional de forma eficiente e garantindo a fitofisionomia da região, ou seja, a Floresta Estacional Semidecidual.

Pela análise de correspondência (CA), foi visto que as toposseqüências Ravina e Plano possuíram maior similaridades florísticas entre si do que as demais.

\section{REFERÊNCIAS}

CITADINI-ZANETTE, V. Fitossociologia e aspectos dinâmicos de um remanescente da Mata Atlantica na microbacia do Rio Novo, Orleans, SC. 1995. 236f. Tese (Doutorado em Ciências) - Universidade Federal de São Carlos, São Carlos, 1995. 
FELFILLI, J. M. Determinação de padrões de distribuição de espécies em uma Mata de Galeria no Brasil Central com a utilização de técnicas de análise multivariada. Boletim do Herbário

Ezechias Paulo Heringer, v.2, p.35-48, 1998.

FINOL, U. H. Nuevos parametros a considerarse en el analisis estrutural de las selvas virgenes tropicales. Revista Forestal Venezolana, v.18, n.12, p.29-42, 1971.

GAMA, J. R. V.; BOTELHO, S. A.; BENTESGAMA, M. M. Composição florística e Estrutura da regeneração natural de floresta secundária de Várzea Baixa no Estuário Amazônico. Revista Árvore, v.26, n.5, p.559-566, 2002.

JARDIM, F. C. S.; HOSOKAWA, R. T. Estrurura da floresta equatorial úmida da Estação Experimental de Silvicultura Tropical do INPA. Acta Amazônica, v.16/17, p.411-508, 1986.

JARDIM, F. C. S.; VOLPATO, M. M. L.; SOUZA, A. L. Dinâmica de sucessão natural com ênfase na sucessão em clareiras. Viçosa, MG: Sociedade de Investigação Florestal, 1993. 60 p. (Documento SIF, 10).

MARANGON, L.C. Florística e fitossociologia de área de floresta estacional semidecidual visando dinâmica de espécies florestais arbóreas no município de Viçosa, MG. 1999. 139f. Tese (Doutorado em Ecologia e Recursos Naturais) - Universidade Federal de São Carlos, São Carlos, 1999.

MARANGON, L. C. Florística arbórea da Mata da Pedreira, Município de Viçosa. Minas Gerais.

Revista Árvore, v.27, n.2, p.207-215, 2003.

MARISCAL FLORES, E. J. Potencial produtivo e alternativas de manejo sustentável de um fragmento de Mata Atlântica secundária, município de Viçosa, Minas Gerais. 1993. 165f. Dissertação (Mestrado em Ciência Florestal) Universidade Federal de Viçosa, Viçosa, MG, 1993.
McCUNE, B.; MEFFORD, M. J. PC-ORD version 4.14: Multivariate analysis of ecological data. Glaneden Beach: $\mathrm{MjM}$ Software Design, 1999. 237p.

MEIRA-NETO, J. A. A. Estudos florísticos, estruturais e ambientais nos estratos arbóreos e herbáceo-arbustivo de uma floresta estacional semidecidual em Viçosa, MG. 1997. 154f. Tese (Doutorado em Biologia) - Universidade Estadual de Campinas, Campinas, 1997.

NEGRELLE, R. R. B. Composição

florística, estrutura fitossociológica e dinâmica de regeneração da floresta Atlântica na reserva Volta Velha, município de Itapoã, SC. 1995. 222 f. Tese (Doutorado em Ciências) - Universidade Federal de São Carlos, São Carlos, 1995.

SOUZA, A. L. et al. Dinâmica da regeneração natural em uma floresta ombrófila densa secundária, após corte de cipós, Reserva Natural da Companhia Vale do Rio Doce S.A., Estado do Espírito Santo, Brasil. Revista Árvore, v.26, n.4, p.411-419, 2002.

UHL, C.; MURPHY, P. G. Composition, estructure, and regeneration of a tierra firme forest in the Amazon basin of Venezuela. Tropical Ecology, v.22, n.2, p.219-237, 1981.

VOLPATO, M. M. L. Regeneração natural em uma floresta secundária no domínio de Mata Atlântica: uma análise fitossociológica. 1994. 123f. Dissertação (Mestrado em Ciência Florestal) Universidade Federal de Viçosa, Viçosa, MG, 1994. 